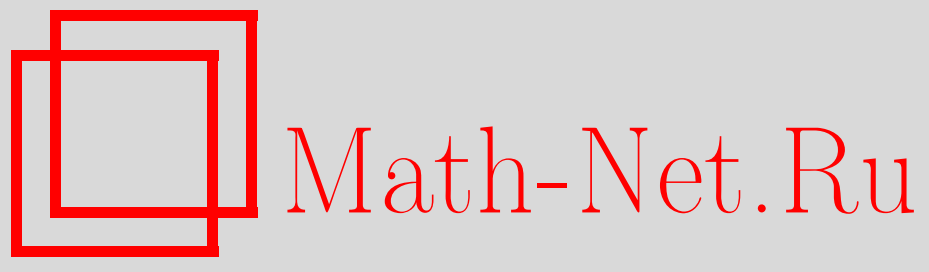

В. Ш. Цагарейшвили, О структуре класса $A(\varphi)$, Матем. заметки, 2006, том 80, выпуск 2, 296-308

DOI: https://doi.org/10.4213/mzm2810

Использование Общероссийского математического портала Math-Net.Ru подразумевает, что вы прочитали и согласны с пользовательским соглашением http: //www.mathnet.ru/rus/agreement

Параметры загрузки:

IP : 35.173 .219 .12

26 апреля 2023 г., 14:41:13

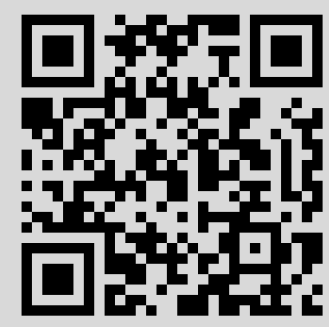




\section{О СТРУКТУРЕ КЛАССА $A(\varphi)$}

\section{В. Цагарейшвили}

В работе решаются вопросы о замкнутости множества $A(\varphi)$ относительно произведения $f \cdot g$, где $f$ и $g$ принадлежат классу $A(\varphi)$. Также изучается вопрос о решении уравнения $B x=C$ (где $B, C \in A(\varphi)$ и $B \neq 0$ ) на множестве $A(\varphi)$.

Библиография: 6 названий.

\section{1. Вспомогательные утверждения и обозначения}

Пусть $\left(\varphi_{n}\right)$ - ортонормированная на $[0,1]$ и полная в $L_{2}(0,1)$ система. Через

$$
c_{n}(f)=\int_{0}^{1} f(x) \varphi_{n}(x) d x, \quad n=1,2, \ldots,
$$

обозначаются коэффициенты Фурье функции $f \in L(0,1)$.

Обозначим через $A(\varphi)$ класс всех функций $f$, для которых сходится ряд

$$
\sum_{n=1}^{\infty}\left|c_{n}(f)\right| \text {. }
$$

Класс $A(\varphi)$ является пространством Банаха с нормой

$$
\|f\|_{A}=\sum_{n=1}^{\infty}\left|c_{n}(f)\right| .
$$

Вопросы замкнутости относительно умножения функций класса $A(\varphi)$ хорошо известны для конкретных ортогональных систем. Для тригонометрической системы $T$ замкнутость класса $A(T)$ относительно умножения функции легко доказывается (см., например, [1]). Обобщение задачи о замкнутости множества $A(T)$ относительно умножения функций рассматривается в работе Ульянова [2]. Ему же принадлежит решение аналогичной задачи относительно системы Хаара (см., например, [3]). Именно, справедлива

Теорема I. Если $f$ и $g$ ограничены и принадлежат классу $A(\chi)$, где $\chi$ - система Xаapa, mo $f \cdot g \in A(\chi)$.

Для изучения вопроса о решении уравнения

$$
B x=C
$$

в пространстве $A(\varphi)$, где $B \in A(\varphi), B(t) \neq 0, t \in[0,1]$, и $C \in A(\varphi)$, является важным результат Винера-Леви (см. [1]).

(C) В. ЦАГАРЕйшвили, 2006 
ТЕОрема II. Если функиия $f \in A(T)$ и если $f$ нигде не обращается в нуль, то $1 / f \in A(T)$, где $T$ - тригонометрическая система.

Наконец, для произвольных полных в $L_{2}(0,1)$ ортонормированных систем можно поставить такие задачи:

1) для каких ортонормированных систем замкнуты относительно умножения функции пространства $A(\varphi)$;

2) для каких ортонормированных систем имеет решение уравнение

$$
B x=C, \quad B, C \in A(\varphi), \quad B \neq 0,
$$

в пространстве $A(\varphi)$.

Пусть множество $E \subset D,|E|>0$, где $D-k$-мерный единичный куб. При $0<p<2$ имеем $^{1}$

$$
\int_{E}\left|\sum_{n=e}^{\infty} a_{n} r_{n}(x)\right|^{p} d x \geqslant C_{p}\left(\sum_{n=q}^{\infty} a_{n}^{2}\right)^{p / 2} .
$$

Здесь $e$ есть $k$-мерный единичный вектор. Вместе с этим $n=\left(n_{1} ; n_{2} ; \ldots ; n_{k}\right), q=$ $\left(q_{1} ; q_{2} ; \ldots ; q_{k}\right)$ и $q$ зависит лишь от $E$; функция $r_{n}(x)=r_{n_{1}}\left(x_{1}\right) \cdot r_{n_{2}}\left(x_{2}\right) \ldots r_{n_{k}}\left(x_{k}\right)$, где $r_{n_{m}}\left(x_{m}\right)$ суть функции Радемахера (см. [5]).

\section{1. Замкнутость.}

Теорема 1. Пусть $\left(\varphi_{n}\right)$ - ортонормированная на $[0,1]$ и полная в $L_{2}(0,1)$ система функиий. Рассмотрим выражение

$$
A_{m n}=\sum_{s=1}^{\infty}\left|\int_{0}^{1} \varphi_{m}(x) \varphi_{n}(x) \varphi_{s}(x) d x\right| .
$$

Для того, чтобы из условий $f \in A(\varphi)$ и $g \in A(\varphi)$ следовало

$$
f \cdot g \in A(\varphi)
$$

необходимо и достаточно, чтобъ

$$
A_{m n}<M, \quad m, n=1,2, \ldots,
$$

где $M$ не зависит от $m$ и $n$. В этом случае

$$
\|f \cdot g\|_{A} \leqslant K\|f\|_{A}\|g\|_{A}, \quad \text { əдe } \quad K=\sup _{m, n} A_{m n} .
$$

ДокаЗАтельство. Достаточность. Пусть $f \in A(\varphi)$ и $g \in A(\varphi)$, тогда почти всюду на $[0,1]$

$$
f(x)=\sum_{m=1}^{\infty} c_{m}(f) \varphi_{m}(x) \quad \text { и } \quad g(x)=\sum_{n=1}^{\infty} c_{n}(g) \varphi_{n}(x) .
$$

\footnotetext{
${ }^{1}$ Неравенство (В) доказывается при помощи неравенства Хинчина-Минковского с использованием метода Бочкарева из работы [4].
} 
Поэтому п.в. на $[0,1]$

$$
f(x) g(x)=\sum_{m=1}^{\infty} \sum_{n=1}^{\infty} c_{m}(f) c_{n}(g) \varphi_{m}(x) \varphi_{n}(x) .
$$

Из (2) имеем

$$
f(x) g(x) \varphi_{s}(x)=\sum_{m=1}^{\infty} \sum_{n=1}^{\infty} c_{m}(f) c_{n}(g) \varphi_{m}(x) \varphi_{n}(x) \varphi_{s}(x) .
$$

В силу полноты в $L_{2}(0,1)$ системы $\left(\varphi_{n}\right)$ и равенства Парсеваля получаем (см. (1))

$$
\begin{aligned}
\int_{0}^{1}\left(\varphi_{n}(x) \varphi_{m}(x)\right)^{2} d x & =\sum_{s=1}^{\infty}\left(\int_{0}^{1} \varphi_{n}(x) \varphi_{m}(x) \varphi_{s}(x) d x\right)^{2} \\
& \leqslant\left(\sum_{s=1}^{\infty}\left|\int_{0}^{1} \varphi_{n}(x) \varphi_{m}(x) \varphi_{s}(x) d x\right|\right)^{2}=A_{m n}^{2}<M^{2}
\end{aligned}
$$

Используя (4), имеем

$$
\begin{aligned}
& \sum_{m=1}^{\infty} \sum_{n=1}^{\infty}\left|c_{m}(f)\right|\left|c_{n}(g)\right| \int_{0}^{1}\left|\varphi_{m}(x) \varphi_{n}(x) \varphi_{s}(x)\right| d x \\
& \quad \leqslant \sum_{m=1}^{\infty}\left|c_{m}(f)\right| \sum_{n=1}^{\infty}\left|c_{n}(g)\right|\left(\int_{0}^{1} \varphi_{s}^{2}(x)\right)^{1 / 2} \cdot\left(\int_{0}^{1}\left(\varphi_{n}(x) \varphi_{m}(x)\right)^{2} d x\right)^{1 / 2} \\
& \leqslant M \sum_{m=1}^{\infty}\left|c_{m}(f)\right| \cdot \sum_{n=1}^{\infty}\left|c_{n}(g)\right|<\infty
\end{aligned}
$$

Следовательно, можно проинтегрировать (3) и получить

$$
c_{s}(f \cdot g)=\sum_{m=1}^{\infty} \sum_{n=1}^{\infty} c_{m}(f) c_{n}(g) \int_{0}^{1} \varphi_{n}(x) \varphi_{m}(x) \varphi_{s}(x) d x .
$$

Наконец, из (5) и (1) следует

$$
\begin{aligned}
\sum_{s=1}^{\infty}\left|c_{s}(f \cdot g)\right| & \leqslant \sum_{m=1}^{\infty} \sum_{n=1}^{\infty}\left|c_{m}(f)\right|\left|c_{n}(g)\right| \sum_{s=1}^{\infty}\left|\int_{0}^{1} \varphi_{n}(x) \varphi_{m}(x) \varphi_{s}(x) d x\right| \\
& =\|f\|_{A} \cdot\|g\|_{A} \cdot A_{m n}<\|f\|_{A} \cdot\|g\|_{A} \cdot M
\end{aligned}
$$

Таким образом, $f \cdot g \in A(\varphi)$ и

$$
\|f \cdot g\|_{A} \leqslant M\|f\|_{A} \cdot\|g\|_{A} .
$$

Необходимость. Рассмотрим несколько случаев.

I) Пусть для некоторых $m_{0}$ и $n_{0}$

$$
A_{m_{0} n_{0}}=+\infty
$$


Поэтому (см. (А))

$$
\sum_{s=1}^{\infty}\left|c_{s}\left(\varphi_{m_{0}} \cdot \varphi_{n_{0}}\right)\right|=\sum_{s=1}^{\infty}\left|\int_{0}^{1} \varphi_{m_{0}}(x) \varphi_{n_{0}}(x) \varphi_{s}(x) d x\right|=A_{m_{0} n_{0}}=+\infty .
$$

Так как $\varphi_{m_{0}} \in A(\varphi), \varphi_{n_{0}} \in A(\varphi)$ и $\varphi_{m_{0}} \cdot \varphi_{n_{0}} \notin A(\varphi)$, то в этом случае справедливость теоремы 1 очевидна.

II) Предположим, что

$$
\varlimsup_{m, n} A_{m n}=+\infty
$$

и для некоторого числа $s_{0}$

$$
\varlimsup_{m, n} \sum_{s=1}^{s_{0}}\left|\int_{0}^{1} \varphi_{m}(x) \varphi_{n}(x) \varphi_{s}(x) d x\right|=+\infty .
$$

Тогда существуют последовательности $\left(m_{k}\right),\left(n_{k}\right)$ и число $s_{1}$ такие, что

$$
\lim _{k \rightarrow \infty}\left|\int_{0}^{1} \varphi_{m_{k}}(x) \varphi_{n_{k}}(x) \varphi_{s_{1}}(x) d x\right|=+\infty .
$$

Предположив $A_{m n}<\infty$ из (4), имеем

$$
\int_{0}^{1}\left(\varphi_{n_{k}}(x) \varphi_{s_{1}}(x)\right)^{2} d x \leqslant A_{n_{k} s_{1}}^{2}<\infty
$$

Рассмотрим последовательность функционалов

$$
G_{k}(f)=\int_{0}^{1} f(x) \varphi_{n_{k}}(x) \varphi_{s_{1}}(x) d x
$$

где $f \in A(\varphi)$.

В силу неравенства Гёльдера и (7) имеем

$$
\begin{aligned}
\left|G_{k}(f)\right| & \leqslant\left(\int_{0}^{1} f^{2}(x) d x\right)^{1 / 2}\left(\int_{0}^{1}\left(\varphi_{n_{k}}(x) \varphi_{s_{1}}(x)\right)^{2} d x\right)^{1 / 2} \\
& \leqslant\|f\|_{L_{2}} \cdot A_{n_{k} s_{1}} \leqslant\|f\|_{A} A_{n_{k} s_{1}} .
\end{aligned}
$$

Таким образом, линейные функционалы (8) непрерывны на $A(\varphi)$.

Теперь рассмотрим последовательность функции $\left(\varphi_{m_{k}}\right)$ из $A(\varphi)$. Согласно $(8)$, (7) и (6) имеем

$$
\left|G_{k}\left(\varphi_{m_{k}}\right)\right|=\left|\int_{0}^{1} \varphi_{m_{k}} \varphi_{n_{k}} \varphi_{s_{1}}(d) d x\right| \text { и } \quad \lim _{k \rightarrow \infty}\left|G_{k}\left(\varphi_{m_{k}}\right)\right|=+\infty .
$$

Так как $\left\|\varphi_{m_{k}}\right\|_{A}=1$, то в силу теоремы Банаха-Штейнгауза существует функция $f_{0} \in A(\varphi)$ такая, что

$$
\varlimsup_{k \rightarrow \infty}\left|G_{k}\left(f_{0}\right)\right|=+\infty .
$$

Отсюда имеем

$$
\varlimsup_{k \rightarrow \infty}\left|C_{n_{k}}\left(f_{0} \cdot \varphi_{s_{1}}\right)\right|=\varlimsup_{k \rightarrow \infty}\left|G_{k}\left(f_{0}\right)\right|=+\infty,
$$


при $f_{0} \in A(\varphi)$ и $\varphi_{s_{1}} \in A(\varphi)$. Теорема 1 и в этом случае доказана.

III) Пусть $\varlimsup_{m, n} A_{m n}=+\infty$. Тогда существуют последовательности $m_{k} \uparrow \infty$ и $n_{k} \uparrow \infty$ такие, что

$$
\lim _{k \rightarrow \infty} A_{m_{k} n_{k}}=+\infty .
$$

Пусть $M_{l}$ выбраны так, чтобы

$$
\sum_{s=M_{l-1}+1}^{M_{l}}\left|\int_{0}^{1} \varphi_{m_{k_{l}}}(x) \varphi_{n_{k_{l}}}(x) \varphi_{s}(x) d x\right|>l^{4}, \quad l=1,2, \ldots, \quad M_{l} \uparrow \infty .
$$

Рассмотрим функции

$$
f_{t}(x)=\sum_{l=1}^{\infty} \frac{1}{l^{2}} \varphi_{m_{k_{l}}}(x) r_{l}(t) \quad \text { и } \quad g_{u}(x)=\sum_{p=1}^{\infty} \frac{1}{p^{2}} \varphi_{n_{k_{p}}}(x) r_{p}(u),
$$

где $\left(r_{l}\right)$ - система Радемахера. Ясно, что $f_{t} \in A(\varphi)$ и $g_{u} \in A(\varphi)$ для любых $t \in[0,1]$ и $u \in[0,1]$. Далее,

$$
f_{t}(x) g_{u}(x)=\sum_{l=1}^{\infty} \sum_{p=1}^{\infty} \frac{1}{l^{2}} \frac{1}{p^{2}} \varphi_{m_{k_{l}}}(x) \varphi_{n_{k_{p}}}(x) r_{l}(t) r_{p}(u)
$$

Отсюда

$$
f_{t}(x) g_{u}(x) \varphi_{s}(x)=\sum_{l=1}^{\infty} \sum_{p=1}^{\infty} \frac{1}{l^{2} p^{2}} \varphi_{m_{k_{l}}}(x) \varphi_{n_{k_{p}}}(x) \varphi_{s}(x) r_{l}(t) r_{p}(u) .
$$

Так как $A_{m n}<\infty$ и

$$
\begin{aligned}
\int_{0}^{1} \mid \varphi_{m_{k_{l}}} & (x) \varphi_{n_{k_{p}}}(x) \varphi_{s}(x) d x \mid \\
& \leqslant\left(\int_{0}^{1} \varphi_{s}^{2}(x) d x\right)^{1 / 2}\left(\int_{0}^{1}\left(\varphi_{m_{k_{l}}}(x) \varphi_{n_{k_{p}}}(x)\right)^{2} d x\right)^{1 / 2}<A_{m n}
\end{aligned}
$$

то из (10) получаем

$$
\begin{aligned}
c_{s}\left(f_{t} \cdot g_{u}\right) & =\int_{0}^{1} f_{t}(x) g_{u}(x) \varphi_{s}(x) d x \\
& =\sum_{l=1}^{\infty} \sum_{p=1}^{\infty} \frac{1}{l^{2} p^{2}} \int_{0}^{1} \varphi_{m_{k_{l}}}(x) \varphi_{n_{k_{p}}}(x) \varphi_{s}(x) d x r_{l}(f) r_{p}(u) .
\end{aligned}
$$

Отсюда, если $E \subset[0,1] \times[0,1]$ - любое множество положительной меры, то, используя неравенство Хинчина (см. (В)), получаем

$$
\begin{aligned}
\iint_{E}\left|c_{s}\left(f_{t} \cdot g_{u}\right)\right| d t d u & \geqslant A(E)\left(\sum_{l=q}^{\infty} \sum_{p=q}^{\infty} \frac{1}{l^{4} p^{4}}\left(\int_{0}^{1} \varphi_{m_{k_{l}}}(x) \varphi_{n_{k_{p}}}(x) \varphi_{s}(x) d x\right)^{2}\right)^{1 / 2} \\
& \geqslant \frac{A(E)}{l^{4}}\left|\int_{0}^{1} \varphi_{m_{k_{l}}}(x) \varphi_{n_{k_{l}}}(x) \varphi_{s}(x) d x\right|
\end{aligned}
$$


где $l>q>0$ - любое целое число, $M_{l-1}<s \leqslant M_{l}$ и $A(E)>0$ зависит лишь от $E$, как и число $q$.

Наконец, из (11) и (9) имеем

$$
\begin{aligned}
& \sum_{s=M_{l-1}+1}^{M_{l}} \iint_{E}\left|c_{s}\left(f_{t} \cdot g_{u}\right)\right| d t d u \\
& \geqslant \frac{A(E)}{l^{4}} \sum_{s=M_{l-1}+1}^{M_{l}}\left|\int_{0}^{1} \varphi_{m_{k_{l}}}(x) \varphi_{n_{k_{l}}}(x) \varphi_{s}(x) d x\right| \geqslant A(E)>0 .
\end{aligned}
$$

Отсюда

$$
\sum_{s=1}^{\infty} \iint_{E}\left|c_{s}\left(f_{t} \cdot g_{u}\right)\right| d t d u=+\infty
$$

Таким образом, для некоторых $t \in[0,1]$ и $u \in[0,1]$ имеем $\left.f_{t} \cdot g_{u} \notin A(\varphi)\right)$. Теорема 1 в случае III доказана.

IV) Пусть для некоторого числа $n_{0}$

$$
\varlimsup_{m} A_{m n_{0}}=+\infty .
$$

Тогда существует последовательность натуральных чисел $\left(m_{k}\right)$ такая, что

$$
\lim _{k \rightarrow \infty} A_{m_{k} n_{0}}=+\infty
$$

Положим, что

$$
f_{t}(x)=\sum_{p=1}^{\infty} \frac{1}{p^{2}} \varphi_{m_{k_{p}}}(x) r_{p}(t),
$$

где $r_{p}(t)$ - функции Радемахера.

Из (12) следует, что для некоторой последовательности $\left(M_{l}\right)$ имеет место неравенство (см. (9))

$$
\sum_{s=M_{l-1}+1}^{M_{l}}\left|\int_{0}^{1} \varphi_{m_{k_{l}}}(x) \varphi_{n_{0}}(x) \varphi_{s}(x) d x\right|>l^{2} .
$$

Далее, имеем

$$
f_{t}(x) \varphi_{n_{0}}(x) \varphi_{s}(x)=\sum_{p=1}^{\infty} \frac{1}{p^{2}} \varphi_{m_{k_{p}}}(x) \varphi_{n_{0}}(x) r_{p}(x) .
$$

Taк как

$$
\begin{aligned}
\int_{0}^{1}\left|\varphi_{m_{k_{l}}}(x) \varphi_{n_{0}}(x) \varphi_{s}(x)\right| d x \leqslant & \left(\int_{0}^{1} \varphi_{s}^{2}(d) d x\right)^{1 / 2} \\
& \times\left(\int_{0}^{1}\left(\varphi_{m_{k_{l}}}(x) \varphi_{n_{0}}(x)\right)^{2} d x\right)^{1 / 2} \leqslant A_{k_{l} n_{0}}<\infty
\end{aligned}
$$

то из (14) получаем

$$
c_{s}\left(f_{t} \varphi_{n_{0}}\right)=\sum_{p=1}^{\infty} \frac{1}{p^{2}} \int_{0}^{1} \varphi_{m_{k_{p}}}(x) \varphi_{n_{0}}(x) \varphi_{s}(x) d x r_{p}(t) .
$$


Отсюда, если $E \subset[0,1]$ - любое множество $(|E|>0)$, то (см. (В))

$$
\begin{aligned}
\int_{E}\left|c_{s}\left(f_{t} \varphi_{n_{0}}\right)\right| d t & \geqslant A(E)\left(\sum_{p=q}^{\infty} \frac{1}{p^{4}}\left(\int_{0}^{1} \varphi_{m_{k_{p}}}(x) \varphi_{n_{0}}(x) \varphi_{s}(x) d x\right)^{2}\right)^{1 / 2} \\
& \geqslant \frac{A(E)}{l^{2}}\left|\int_{0}^{1} \varphi_{m_{k_{l}}}(x) \varphi_{n_{0}}(x) \varphi_{s}(x) d x\right|
\end{aligned}
$$

где $l>q>0$ - любое число и $M_{l-1}<s<M_{l}$. Следовательно (см. (13)),

$$
\begin{aligned}
\sum_{s=M_{l-1}+1}^{M_{l}} \int_{E} c_{s}\left(f_{t} \varphi_{n_{0}}\right) \mid d t & \geqslant \frac{A(E)}{l^{2}} \sum_{s=M_{l-1}+1}^{M_{l}}\left|\int_{0}^{1} \varphi_{m_{k_{l}}}(x) \varphi_{n_{0}}(x) \varphi_{s}(x) d x\right| \\
& \geqslant \frac{A(E)}{l^{2}} \cdot l^{2}=A(E)>0 .
\end{aligned}
$$

Отсюда

$$
\sum_{s=1}^{\infty} \int_{E}\left|c_{s}\left(f_{t} \varphi_{n_{0}}\right)\right| d t=+\infty
$$

Таким образом, с одной стороны, имеем $f_{t} \in A(\varphi)$ (для любого $\left.t \in[0,1]\right)$ и $\varphi_{n_{0}} \in$ $A(\varphi)$. С другой стороны, для некоторых $t \in[0,1]$ следует

$$
\sum_{s=1}^{\infty}\left|c_{s}\left(f_{t} \varphi_{n_{0}}\right)\right|=+\infty
$$

т.е. $f_{t} \cdot \varphi_{n_{0}} \notin A(\varphi)$. Теорема полностью доказана.

1.2. Вопросы эффективности. Если $\left(\varphi_{n}\right)$ - тригонометрическая система или система Уолша, тогда

$$
A_{m n}=\sum_{s=1}^{\infty}\left|\int_{0}^{1} \varphi_{m}(x) \varphi_{n}(x) \varphi_{s}(x) d x\right|<1 .
$$

Для системы Хаapa

$$
\varlimsup_{m, n} A_{m n}=+\infty
$$

В самом деле,

$$
\int_{0}^{1} \chi_{n}^{(1)}(x) \chi_{n}^{(1)}(x) \chi_{n-1}^{(1)}(x) d x=2^{n} \int_{0}^{2^{-n}} \chi_{n-1}^{(1)}(x) d x=2^{(n-1) / 2} .
$$

Таким образом,

$$
A_{2^{n}+12^{n}+1} \geqslant 2^{(n-1) / 2} \text {. }
$$

Следовательно, если $\chi$ - система Хаара, то $A(\chi)$ не замкнуто относительно умножения функций из $A(\chi)$. 
2. Решение уравнения $B x=C$

Пусть $B \in A(\varphi)$ и $C \in A(\varphi)$, причем $B(t) \neq 0, t \in[0,1]$. Введем обозначение

$$
B_{k}(f)=\sum_{m=1}^{\infty}\left|\int_{0}^{1} \frac{1}{f(t)} \varphi_{k}(t) \varphi_{m}(t) d t\right|,
$$

где $f \in L(0,1)$ - любая функция такая, что $f(t) \neq 0$ при $t \in[0,1]$.

ТеОрема 2. Для того чтобы уравнение

$$
B x=C
$$

имело решение в $A(\varphi)$, необходимо и достаточно, чтоби для любой функиии $f \in$ $A(\varphi), f \neq 0$, имело место неравенство

$$
B_{k}(f)<M(f), \quad k=1,2, \ldots,
$$

где $M(f)>0$ не зависит от $k$.

Доказательство. Достаточность. Пусть $C(t) \in A(\varphi)$ и $B(t) \in A(\varphi), B(t) \neq 0$, $t \in[0,1]$. Тогда п.в. на $[0,1]$

$$
C(t)=\sum_{k=1}^{\infty} b_{k}(C) \varphi_{k}(t)
$$

где

$$
b_{k}(C)=\int_{0}^{1} C(t) \varphi_{k}(t) d t
$$

Из (15) следует, что

$$
x(t)=\frac{C(t)}{B(t)} .
$$

Поэтому из (16) получаем

$$
x(t)=\frac{C(t)}{B(t)}=\sum_{k=1}^{\infty} b_{k}(C) \frac{1}{B(t)} \varphi_{k}(t) .
$$

В силу полноты системы $\left(\varphi_{n}\right)$, применяя неравенство Парсеваля и неравенство $B_{k}(f)<M(f)$, имеем

$$
\begin{aligned}
\int_{0}^{1}\left(\frac{1}{f(t)} \varphi_{m}(t)\right)^{2} d t & =\sum_{s=1}^{\infty}\left(\int_{0}^{1} \frac{1}{f(t)} \varphi_{m}(t) \varphi_{s}(t) d t\right)^{2} \\
& \leqslant\left(\sum_{s=1}^{\infty}\left|\int_{0}^{1} \frac{1}{f(t)} \varphi_{m}(t) \varphi_{s}(t) d t\right|\right)^{2} \leqslant B_{m}^{2}(f)<M^{2}(f) .
\end{aligned}
$$

Используя (18), получаем

$$
\begin{aligned}
\sum_{k=1}^{\infty}\left|b_{k}(C)\right| & \left|\int_{0}^{1} \frac{1}{B(t)} \varphi_{k}(t) \varphi_{s}(t) d t\right| \\
& \leqslant \sum_{k=1}^{\infty}\left|b_{k}(C)\right|\left(\int_{0}^{1} \varphi_{s}^{2}(t) d t\right)^{1 / 2}\left(\int_{0}^{1}\left(\frac{1}{B(t)} \varphi_{k}(t)\right)^{2} d t\right)^{1 / 2} \leqslant M(B)\|C\|_{A} .
\end{aligned}
$$


Из (17) имеем

$$
x(t) \varphi_{m}(t)=\sum_{k=1}^{\infty} b_{k}(c) \frac{1}{B(t)} \varphi_{k}(t) \varphi_{m}(t) .
$$

Поэтому, учитывая (19), получим

$$
b_{m}(x)=\int_{0}^{1} x(t) \varphi_{m}(t) d t=\sum_{k=1}^{\infty} b_{k}(C) \int_{0}^{1} \frac{1}{B(t)} \varphi_{k}(t) \varphi_{m}(t) d t
$$

Отсюда

$$
\begin{aligned}
\sum_{m=1}^{\infty}\left|b_{m}(x)\right| & \leqslant \sum_{k=1}^{\infty} b_{k}(C)\left|\sum_{m=1}^{\infty}\right| \int_{0}^{1} \frac{1}{B(t)} \varphi_{k}(t) \varphi_{m}(t) d t \mid \\
& =\sum_{k=1}^{\infty}\left|b_{k}(C)\right|\left|B_{k}(B)\right| \leqslant M(B)\|C\|_{A} .
\end{aligned}
$$

Достаточность теоремы доказана.

Необходимость. Пусть для некоторой функции $B_{0} \in A(\varphi)$ и $B_{0}(t) \neq 0$ при $t \in[0,1]$

$$
\varlimsup_{k \rightarrow \infty} B_{k}\left(B_{0}\right)=+\infty .
$$

Поэтому для некоторой подпоследовательности $k_{n} \uparrow \infty$ имеется, что

$$
\lim _{n \rightarrow \infty} B_{k_{n}}\left(B_{0}\right)=+\infty
$$

Следовательно, существует последовательность $N_{l} \uparrow \infty$ такая, что

$$
\sum_{m=N_{l-1}+1}^{N_{l}}\left|\int_{0}^{1} \frac{1}{B_{0}(t)} \varphi_{k_{n_{l}}}(t) \varphi_{m}(t) d t\right|>l^{2}, \quad l=1,2, \ldots
$$

Предположим, что

$$
C_{x}(t)=\sum_{l=1}^{\infty} \frac{1}{l^{2}} \varphi_{n_{k_{l}}}(t) r_{l}(x)
$$

Очевидно, что $C_{x} \in A(\varphi)$ для любого $x \in[0,1]$.

Рассмотрим уравнение

$$
B_{0}(t) \cdot X(t)=C_{x}(t)
$$

Отсюда

$$
X_{x}(t)=\frac{C_{x}(t)}{B_{0}(t)}
$$

Тогда

$$
X_{x}(t) \varphi_{k}(t)=\sum_{l=1}^{\infty} \frac{1}{l^{2}} \frac{1}{B_{0}(t)} \varphi_{k_{n_{l}}}(t) r_{l}(x)
$$

Так как (см. (18))

$$
\int_{0}^{1}\left(\frac{1}{B_{0}(t)} \varphi_{k_{n_{l}}}\right)^{2} \leqslant B_{k_{n_{l}}}^{2}(f)<\infty
$$


TO

$$
b_{k}\left(X_{x}\right)=\int_{0}^{1} X_{x}(t) \varphi_{k}(t) d t=\sum_{l=1}^{\infty} \frac{1}{l^{2}} \int_{0}^{1} \frac{1}{B_{0}(t)} \varphi_{k_{n_{l}}}(t) \varphi_{k}(t) d t r_{l}(x) .
$$

Пусть $F \subset[0,1]$ - любое множество и $|F|>0$, тогда (см. (В))

$$
\begin{aligned}
\int_{F}\left|b_{k}\left(X_{x}\right)\right| d x & \geqslant A(F)\left(\sum_{l=q}^{\infty} \frac{1}{l^{4}}\left(\int_{0}^{1} \frac{1}{B_{0}(t)} \varphi_{k_{n_{l}}}(t) \varphi_{k}(t) d t\right)^{2}\right)^{1 / 2} \\
& \geqslant A(F) \cdot \frac{1}{l^{2}}\left|\int_{0}^{1} \frac{1}{B_{0}(t)} \varphi_{k_{n_{l}}}(t) \varphi_{k}(t) d t\right|
\end{aligned}
$$

где $N_{l-1}<k<N_{l}, A(F)>0$ не зависит от $k$ и $l>q>0$ - любое целое число.

Таким образом,

$$
\begin{aligned}
\sum_{k=1}^{\infty} \int_{F}\left|b_{k}\left(X_{x}\right)\right| d x & \geqslant A(F) \sum_{l=1}^{\infty} \sum_{k=N_{l-1}+1}^{N_{l}} \frac{1}{l^{2}}\left|\int_{0}^{1} \frac{1}{B_{0}(t)} \varphi_{k_{n_{l}}}(t) \varphi_{k}(t) d t\right| \\
& \geqslant A(F) \sum_{l=q}^{\infty} \frac{1}{l^{2}} l^{2}=+\infty
\end{aligned}
$$

т.е. для п.в. $x \in[0,1] \quad X_{x} \notin A(\varphi)$. Теорема доказана.

ЗАмЕчАНИЕ 1 . Если $B_{k_{0}}\left(B_{0}\right)=+\infty$ для некоторого $k_{0}$, то

$$
\sum_{m=1}^{\infty}\left|\int_{0}^{1} \frac{\varphi_{k_{0}}(t)}{B_{0}(t)} \varphi_{m}(t) d t\right|=\sum_{m=1}^{\infty}\left|b_{m}\left(\frac{\varphi_{k_{0}}}{B_{0}}\right)\right|=B_{k_{0}}\left(B_{0}\right)=+\infty .
$$

Отсюда следует, что уравнение (при $B_{0} \in A(\varphi)$ и $\varphi_{k_{0}} \in A(\varphi)$ )

$$
B_{0} \cdot X=\varphi_{k_{0}}
$$

не имеет решения в $A(\varphi)$.

ЗАмЕчАНИЕ 2. В силу теоремы II для тригонометрической системы

$$
B_{k}(f)<M(f), \quad k=1,2, \ldots,
$$

для любой функции $f \in A(\varphi), f \neq 0$.

ЗАмЕчАниЕ 3. В случае системы Хаара

$$
\varlimsup_{k} B_{k}(B)=+\infty
$$

для некоторой функции $B \in A(\chi)$.

В самом деле, положим

$$
B(t)= \begin{cases}t^{1 / 2} & \text { при } t \in(0,1] \\ 1 & \text { при } t=0\end{cases}
$$

Отсюда

$$
\int_{0}^{1}\left|B^{\prime}(t)\right| d t=\frac{1}{2} \int_{0}^{1} t^{-1 / 2} d t=\frac{1}{2} \cdot 2=1
$$


Значит, $B$ есть функция с конечным изменением. Поэтому (см. [6])

$$
B(t) \in A(\chi) .
$$

С другой стороны,

$$
\int_{0}^{1} \chi_{n}^{(1)}(t) \chi_{n}^{(1)}(t) \cdot B^{-1}(t) d t=2^{n} \int_{0}^{2^{-n}} t^{-1 / 2} d t=2^{n / 2+1} .
$$

Следовательно,

$$
B_{2^{n}+1}(B)>2^{n / 2} \quad \text { и } \quad \varlimsup_{n \rightarrow \infty} B_{2^{n}+1}(B)=+\infty .
$$

Таким образом, при $B \in A(\chi)$ и $C \in A(\chi)$ уравнение $B X=C$ не имеет решения в пространстве $A(\chi)$.

ЗАмечание 4 . В теореме 2 нельзя опустить условие $f \in A(\varphi)$ при $B_{k}(f)<M(f)$. В самом деле, пусть $\left(\varphi_{n}\right)$ - ортонормированная система, полная в $L_{2}(0,1)$ и

$$
\sum_{n=1}^{\infty}\left|\int_{0}^{1} \varphi_{n}(x) d x\right|=+\infty
$$

Тогда $1 \notin A(\varphi)$, но

$$
B_{k}(1)=\sum_{n=1}^{\infty}\left|\int_{0}^{1} \frac{1}{1} \varphi_{k}(x) \varphi_{m}(x) d x\right|=1 .
$$

Теперь, если $B(x) \in A(\varphi)$ - некоторая функция $(B \neq 0)$, то уравнение

$$
B(x) X(x)=B(x)
$$

не имеет решения в $A(\varphi)$, так как $1 \notin A(\varphi)$.

\section{3. Критерии абсолютной сходимости}

Пусть $\left(\varphi_{n}\right)$ - ортонормированная на $[0,1]$ и полная в $L_{2}(0,1)$ система функций. Рассмотрим выражение

$$
K_{n}(f, g)=\sum_{s=1}^{\infty}\left|\int_{0}^{1} \frac{f(x)}{g(x)} \varphi_{n}(x) \varphi_{s}(x) d x\right|,
$$

где $f \neq 0$ и $g \neq 0$ при $x \in[0,1]$.

Теорема 3. Для того чтобы имело место условие

$$
f \in A(\varphi),
$$

необходимо и достаточно, чтобы для некоторого $g \in A(\varphi)$ соблюдалось неравенство

$$
K_{n}(f, g)<M(f, g), \quad n=1,2, \ldots,
$$

где $M(f, g)$ не зависит от $n$. 
ДокАЗАтЕЛЬСтво. Достаточность. Пусть $g \in A(\varphi)$, тогда

$$
g(x)=\sum_{n=1}^{\infty} c_{n}(g) \varphi_{n}(x)
$$

п.в. на $[0,1]$, где

$$
c_{n}(g)=\int_{0}^{1} g(x) \varphi_{n}(x) d x
$$

Так как

$$
f(x) g(x)=\sum_{n=1}^{\infty} c_{n}(g) \varphi_{n}(x) f(x)
$$

то

$$
f(x)=\sum_{n=1}^{\infty} c_{n}(g) \frac{f(x)}{g(x)} \varphi_{n}(x) .
$$

Следовательно,

$$
f(x) \varphi_{s}(x)=\sum_{n=1}^{\infty} c_{n}(g) \frac{f(x)}{g(x)} \varphi_{n}(x) \varphi_{s}(x) .
$$

В силу полноты $\left(\varphi_{n}\right)$

$$
\begin{aligned}
\int_{0}^{1}\left(\frac{f(x)}{g(x)} \varphi_{n}(x)\right)^{2} d x & \leqslant \sum_{s=1}^{\infty}\left(\int_{0}^{1} \frac{f(x)}{g(x)} \varphi_{n}(x) \varphi_{s}(x) d x\right)^{2} \\
& \leqslant\left(\sum_{s=1}^{\infty}\left|\int_{0}^{1} \frac{f(x)}{g(x)} \varphi_{n}(x) \varphi_{s}(x) d x\right|\right)^{2} \\
& =K_{n}^{2}(f, g) \leqslant M^{2}(f, g) .
\end{aligned}
$$

Стало быть, функция (20) интегрируема потому, что

$$
\begin{aligned}
\sum_{n=1}^{\infty}\left|c_{n}(g)\right| & \int_{0}^{1}\left|\frac{f(x)}{g(x)} \varphi_{n}(x) \varphi_{s}(x)\right| d x \\
& \leqslant \sum_{n=1}^{\infty}\left|c_{n}(g)\right|\left(\int_{0}^{1}\left(\frac{f(x)}{g(x)} \varphi_{s}(x)\right)^{2} d x\right)^{1 / 2} \cdot\left(\int_{0}^{1} \varphi_{n}^{2} d x\right)^{1 / 2} \\
& \leqslant M(f, g)\|g\|_{A} .
\end{aligned}
$$

Таким образом,

$$
c_{s}(f)=\int_{0}^{1} f(x) \varphi_{s}(x) d x=\sum_{n=1}^{\infty} c_{n}(g) \int_{0}^{1} \frac{f(x)}{g(x)} \varphi_{n}(x) \varphi_{s}(x) d x .
$$

Отсюда

$$
\begin{aligned}
\sum_{s=1}^{\infty}\left|c_{s}(f)\right| & \leqslant \sum_{n=1}^{\infty}\left|c_{n}(g)\right| \sum_{s=1}^{\infty}\left|\int_{0}^{1} \frac{f(x)}{g(x)} \varphi_{n}(x) \varphi_{s}(x) d x\right| \\
& \leqslant \sum_{n=1}^{\infty}\left|c_{n}(g)\right| \cdot K_{n}(f, g) \leqslant M(f, g)\|g\|_{A} .
\end{aligned}
$$


Следовательно, $f \in A(\varphi)$ и $\|f\|_{A} \leqslant M(f, g)\|g\|_{A}$.

Необходимость. Пусть для некоторой функции $f_{0}, f_{0} \neq 0$, и для любого $g \in A(\varphi)$ имеем

$$
\varlimsup_{n} K_{n}\left(f_{0}, g\right)=+\infty .
$$

Допустим противное и предположим, что $f_{0} \in A(\varphi)$. Так как $g \in A(\varphi)$ - любая функция и $f_{0} \neq 0$, то в качестве $g$ мы можем взять функцию $f_{0}$.

Таким образом, при $f_{0} \in A(\varphi)$ имеем

$$
K_{n}\left(f_{0}, f_{0}\right)=\sum_{s=1}^{\infty}\left|\int_{0}^{1} \frac{f_{0}(x)}{f_{0}(x)} \varphi_{n}(x) \varphi_{s}(x) d x\right|=\sum_{s=1}^{\infty}\left|\int_{0}^{1} \varphi_{n}(x) \varphi_{s}(x) d x\right|=1 .
$$

Поэтому

$$
\lim _{k \rightarrow \infty} K_{n}\left(f_{0}, f_{0}\right)=1,
$$

что и противоречит условию (21). Теорема полностью доказана.

\section{СПИСОК ЦИТИРОВАННОЙ ЛИТЕРАТУРЫ}

[1] Ж.П. Кахан, Абсолютно сходящиеся ряды Фуръе, Мир, М., 1976.

[2] П. Л. Ульянов, "Об абсолютной сходимости тригонометрических рядов Фурье”, Докл. АН СCCP, 322:2 (1992), 253-258.

[3] В. Бугадзе, Рядъ Фуръе-Хаара суперпозиций функиий, Тбилиси, 1988.

[4] С. В. Бочкарев, "Абсолютная сходимость рядов Фурье относительно общих ортогональных полных систем", УМН, 27:1 (1972), 53-76.

[5] В. Цагарейшвили, Классы функиии и коэффициенты Фуръе, Докторская диссертация, Тбилиси, 2004.

[6] П. Л. Ульянов, “О рядах по системе Хаара”, Матем. сб., 63(105):3 (1964), 356-391.

\section{В. Цагарейшвили}

